\title{
Prognostic significance of PD-L1 protein expression and copy number gains in locally advanced cervical cancer
}

\author{
Kongsak Loharamtaweethong, ${ }^{1}$ Chalermpak Supakatitham, ${ }^{1}$ Songkhun Vinyuvat, ${ }^{2}$ Napaporn Puripat, ${ }^{1}$ \\ Sujitra Tanvanich, ${ }^{1}$ Unaporn Sitthivilai ${ }^{1}$
}

\begin{abstract}
Background: Although immune checkpoint inhibitors against programmed death-1 (PD-1) and its ligand (PD-L1) have demonstrated promising results in several solid malignancies, including cervical cancer, there are some limitations to using PD-L1 immunohistochemical expression as a predictive biomarker for selecting patients who may benefit from such therapy.
\end{abstract}

Objective: To examine the protein expression and genetic status of PD-L1 with clinical outcomes in locally advanced cervical cancer.

Methods: We investigated the PD-L1 gene copy number gains assessed by fluorescence in situ hybridization (FISH) and PD-L1 expression using immunohistochemistry in 123 patients with locally advanced cervical cancers between December 2008 and December 2016.

Results: The prevalence of PD-L1 immunohistochemical expression was detected in 103/123(83\%) cases. PD-L1 gene amplification and polysomy were detected in $7 \%$ and $40 \%$ of cases, respectively. PD-L1 gene amplification and polysomy were associated with positive PD-L1 immunostaining (score $1+$ to $3+$ ) in $88 \%$ and $68 \%$ of cases, respectively. Clinically, PD-L1 immunopositivity was associated with parametrial invasion at diagnosis. In contrast, PD-L1 polysomy was associated with parametrial invasion and FIGO stages III-IV, whereas PD-L1 amplification was associated with nodal metastasis. In multivariate analysis, PD-L1 amplification was predictive of worse RFS (HR, 5.68; 95\%CI, 1.98$16.28 ; p=0.001$ ), whereas PD-L1 polysomy was predictive of worse LRR (HR, 4.13; 95\%CI, 1.63-10.49; $p=0.003$ ). PDL1 immunohistochemical expression was not associated with worse outcomes in Cox models.

Conclusions: Our results showed that an increase in PD-L1 gene copy number could be a novel prognostic and possible predictive biomarker for anti-PD-1/PD-L1 therapy in locally advanced cervical cancer.

Key words: Prognosis, programmed cell death-ligand 1, protein expression, copy number status, cervical cancer

\section{Affiliations:}

Department of Anatomical Pathology, Faculty of Medicine, Vajira Hospital, Navamindradhiraj University, Bangkok, Thailand

Department of Medical Services, Institute of Pathology, Ministry of Public Health, Bangkok, Thailand

\section{Introduction}

Cervical cancer is the second most common cancer in women worldwide, particularly in developing countries. ${ }^{1}$ Most patients present with locally advanced stages ${ }^{2}$ defined as stages IB2-IVA by the International Federation of Gynaecology and Obstetrics (FIGO), and concurrent chemoradiation remains the standard of treatment for these patients. However, the majority of recurrences occur within two years after treatment..$^{2-4}$

\author{
Corresponding author: \\ Kongsak Loharamtaweethong \\ Department of Anatomical Pathology, Faculty of Medicine, \\ Vajira Hospital, Navamindradhiraj University, \\ 681 Samsen Road, Dusit, Bangkok, 10300 Thailand \\ E-mail: Kongsakloharamtaweethong@hotmail.com
}

Most patients who experience recurrence still have a poor prognosis. In addition, a molecular predictor of tumour recurrence after concurrent chemoradiotherapy is lacking. If patients who are at risk for recurrence could be effectively classified, then more specific treatments might have great benefit to these patients. Potentially, identification of cervical cancer with effective molecular biomarkers could be helpful for determining prognosis. 
Theoretically, tumours can evade immune surveillance by upregulating programmed cell death-ligand 1 (PD-L1) expression. PD-L1 is known to play a key role in the inhibition of the $\mathrm{T}$ cell-mediated immune response, leading to the progression of tumours. PD-L1 can interact with programmed cell death 1 (PD-1), an inhibitory immune checkpoint receptor that plays a major role in immune evasion during tumour progression, ${ }^{5}$ leading to the inactivation of $\mathrm{T}$ cells via the inhibition of T-cell receptor signalling and co-stimulatory signals..$^{6-9}$

PD-L1 overexpression has been identified in many solid cancers, ${ }^{10}$ such as malignant melanoma, ${ }^{11}$ pulmonary cancer ${ }^{12}$ and colorectal cancer. ${ }^{13}$ However, the relationship between PD-L1 expression levels and overall disease prognosis is still inconclusive. Wu et al. ${ }^{10}$ demonstrated that PD-L1 overexpression is related to worse overall survival in gastric carcinoma, hepatocellular carcinoma, oesophageal carcinoma, and transitional cell carcinoma, whereas this relationship was not observed in pulmonary cancer and malignant melanoma.

In addition, PD-L1 immunohistochemical expression on the surfaces of tumour cells could show some limitations for identifying patients who may benefit from immune checkpoint inhibitors, such as heterogeneous expression and its dynamic expression.

Interestingly, in nodular sclerosing Hodgkin lymphoma and primary mediastinal large B-cell lymphoma, 9p24.1 gene amplification has recently been demonstrated to be a key mechanism for PD-L1 protein overexpression. ${ }^{14}$ Accordingly, in a subset of carcinomas in the stomach as well as the colon, triple-negative breast cancers and glioblastomas, the 9p24.1 gene mechanism has been discovered. ${ }^{15,16}$ More recently, cervical and vulvar squamous cell carcinomas showed the genetic origin of amplified PD-L1 expression as well. In 67\% of cervical and $43 \%$ of vulvar squamous cell carcinomas assessed using FISH, the genes encoding PD-L1 and PD-L2, CD274 and PDCD1LG2, were co-amplified or could be found on multiple copies of the same chromosome. ${ }^{17}$ As a result, 9p24.1 gene copy number changes are an essential mechanism of increased PD-L1 expression in cervical squamous cell carcinomas, as proposed by the data. However, this study did not investigate the correlation of genetic changes with clinical outcomes.

Hence, the present study aimed to assess the pretreatment of PD-L1 protein and gene expression in patients with locally advanced cervical carcinoma. We investigated the correlation between the frequency of copy number gains of the PD-L1 gene and the upregulation of the corresponding proteins and determined its prognostic impact using a clinically well-characterized cohort comprising 123 patients with cervical cancer.

\section{Materials and Methods}

The retrospective cohort consisted of cervical cancer patients with FIGO stage IB2-IVA who underwent tissue biopsies of squamous cell carcinoma and adenocarcinoma of the cervix between December 2008 and December 2016 at the Faculty of Medicine, Navamindradhiraj University. The study was approved by the Institutional Review Board of the Faculty of Medicine, Navamindradhiraj University. H\&E-stained sections were reviewed by two pathologists (KL, CS). Complete clinicopathologic data were available for all patients.

\section{Immunohistochemistry}

Immunohistochemistry was carried out in all cases using a monoclonal antibody recognizing PD-L1 (clone SP263, Ventana Medical Systems, Inc., Tucson, AZ, USA) and performed on a systematized staining platform (Benchmark ULTRA; Ventana Medical Systems, Inc., Tucson, AZ, USA). Four-micron whole tissue sections were cut and stained. An OptiView DAB IHC Detection Kit (Ventana Medical Systems, Inc., Tucson, AZ, USA) was used according to the manufacturer's guidelines for the visualization of the primary anti-PD-L1 antibody. For all immunohistochemical staining reactions, human placental tissue was used as a positive control. The staining of PD-L1 in tissues was quantified using two scoring systems as follows: (1) intensity scores: IHC 0 specified no appreciable staining in cancer cells or staining in under $10 \%$ of cancer cells; IHC 1+ specified slightly noticeable partial membrane staining in $>10 \%$ of cancer cells; IHC $2+$ specified moderate staining of the whole membrane in $>10 \%$ of cancer cells; and IHC 3+ specified intense staining of the whole membrane in $>10 \%$ of cancer cells; (2) modified histochemical scores (H-scores): the modified $\mathrm{H}$-score was calculated by multiplying the average membranous intensity score $(0$, absent; 1 , weak; 2 , moderate; 3 , strong) by the percentage of stained cells (from $0 \%$ to $100 \%$ ). Consequently, the $\mathrm{H}$-score could range from 0 to 300 . These scoring systems were also used in previous studies. ${ }^{17,18}$

Tumours with $10 \%$ or more cells exhibiting the PD-L1 immunohistochemical reaction, irrespective of intensity, were defined as PD-L1 positive because a previous study had established this cut-off value using the same antibody clone. ${ }^{19}$

\section{PD-L1 fluorescence in situ hybridization}

Tissue microarrays (TMAs) with $3 \mathrm{~mm}$ core diameter were obtained from representative cervical cancer tissues. A minimum of 2 and up to 4 tumour cores from the tumour invasion front and/or the tumour centre were taken from the tumours in areas previously marked by two pathologists (KL, CS). All TMA cores were validated to contain a sufficient number of tumour cells by reviewing haematoxylin and eosin (HE)-stained sections. Dual-colour FISH analysis was performed on $4 \mu \mathrm{m}$ FFPE TMA sections. A SPEC CD274, PDCD1LG2/CEN9 Dual Colour Probe (Zytovision, Bremerhaven, Germany) was used according to the manufacturer's guidelines. After screening the entire area of individual cores, the probe signals from a monolayer of at least 50 tumour cell nuclei were counted at $\times 100$ magnification in at least five representative images per case. As previously described, ${ }^{20} \mathrm{PD}-\mathrm{L} 1$ amplification was identified if PD-L1/CEP9 ratio $\geq 2.0$, while polysomy was identified if the median copy number of the PD-L1 gene $\geq 3.0$ and the ratio of this value to the CEP9 signals was $<2.0$. All other tumours were deemed to display disomy.

\section{Statistical analysis}

Statistical analysis was performed using Stata Statistical Software (College Station, TX: StataCorp LP; http://www.stata. com). The distribution of qualitative data was compared between groups using a $\chi^{2}$-test or Fisher's exact test, depending on the cell counts of the corresponding contingency tables. 
For survival analysis, the Kaplan-Meier method was used to compute recurrence-free survival (RFS), cancer-specific survival (CSS), and locoregional-recurrence-free survival (LRR). Univariate and multivariate analyses were performed using the Cox proportional hazards model, and the differences between groups were analysed by a log-rank test. For all statistical analyses, $p<0.05$ was considered statistically significant.

\section{Results}

Clinicopathological significance of PD-L1 expression and PD-L1 copy number status in locally advanced cervical cancer

The clinicopathological characteristics of the cervical cancer patients in relation to PD-L1 immunohistochemical expression and PD-L1 copy number status are shown in Tables 1 and 2, respectively.

Table 1. Clinicopathological data according to PD-L1 immunohistochemical expression.

\begin{tabular}{|c|c|c|c|}
\hline $\begin{array}{c}\text { Clinicopathologic } \\
\text { Parameters }\end{array}$ & PD-L1 positive & PD-L1 negative & $p$-value \\
\hline $\mathrm{n}(\%)$ & $103(83)$ & $20(17)$ & \\
\hline Age (years) & $55.39 \pm 13.18$ & $53.90 \pm 9.82$ & 0.346 \\
\hline$<60$ & $66(64)$ & $15(75)$ & \\
\hline$\geq 60$ & $37(36)$ & $5(25)$ & \\
\hline Histology & & & $<0.001^{*}$ \\
\hline SCC & $97(94)$ & $13(65)$ & \\
\hline Adeno & $6(6)$ & $7(35)$ & \\
\hline Tumor Size $(\mathrm{cm})$ & & & 0.076 \\
\hline$<4$ & $22(21)$ & $8(40)$ & \\
\hline$\geq 4$ & $81(79)$ & $12(60)$ & \\
\hline
\end{tabular}

\begin{tabular}{lccc}
$\begin{array}{l}\text { Clinicopathologic } \\
\text { Parameters }\end{array}$ & PD-L1 positive & PD-L1 negative & p-value \\
Staging & $61(59)$ & $16(80)$ & 0.079 \\
Stage IB2-IIB & $42(41)$ & $4(20)$ & \\
Stage IIIA-IVA & $15(15)$ & $10(50)$ & $<0.001^{*}$ \\
Parametrial invasion & $88(85)$ & $10(50)$ & 0.617 \\
No & & & \\
Yes & $77(75)$ & $16(80)$ & \\
Lymph node positive & $26(25)$ & $4(20)$ & \\
\hline No & & & \\
\hline Yes & & & \\
Treatment & $99(96)$ & $20(100)$ & \\
CTRT & & & \\
Radical RT & & & \\
\hline
\end{tabular}

Values are presented as number (\%), mean \pm standard deviation.

Abbreviations: SCC, squamous cell carcinoma; Adeno, adenocarcinoma; CTRT, concurrent chemoradiotherapy; RT, radiation therapy. ${ }^{*} p$-value $<0.05$, Statistically significant.

Table 2. Clinicopathological data according to PD-L1 copy number status.

\begin{tabular}{|c|c|c|c|c|c|}
\hline $\begin{array}{l}\text { Clinicopathologic } \\
\text { Parameters }\end{array}$ & $\begin{array}{c}\text { PD-L } \\
\text { amplification }\end{array}$ & $\begin{array}{l}\text { PD-L1 } \\
\text { polysomy }\end{array}$ & $\begin{array}{l}\text { PD-L1 } \\
\text { disomy }\end{array}$ & $p$-value ${ }^{\mathrm{a}}$ & $p$-value ${ }^{\mathrm{b}}$ \\
\hline n (\%) & $8(7)$ & $50(40)$ & $65(53)$ & & \\
\hline Age (years) & $56.5 \pm 14.51$ & $55.02 \pm 13.12$ & $55.07 \pm 12.29$ & 0.912 & 0.879 \\
\hline$<60$ & $5(63)$ & $33(66)$ & $42(64)$ & & \\
\hline$\geq 60$ & $3(37)$ & $17(34)$ & $23(36)$ & & \\
\hline Histology & & & & 0.370 & 0.423 \\
\hline SCC & $8(100)$ & $43(86)$ & $59(91)$ & & \\
\hline Adeno & $0(0)$ & $7(14)$ & $6(9)$ & & \\
\hline Tumor Size $(\mathrm{cm})$ & & & & 0.057 & 0.083 \\
\hline$<4$ & $0(0)$ & $9(18)$ & $21(32)$ & & \\
\hline$\geq 4$ & $8(100)$ & $41(82)$ & $44(68)$ & & \\
\hline Staging & & & & 0.314 & $<0.001^{*}$ \\
\hline Stage IB2-IIB & $5(63)$ & $21(42)$ & $51(78)$ & & \\
\hline Stage IIIA-IVA & $3(37)$ & $29(58)$ & $14(22)$ & & \\
\hline
\end{tabular}


Table 2. (Continued)

\begin{tabular}{|c|c|c|c|c|c|}
\hline $\begin{array}{l}\text { Clinicopathologic } \\
\text { Parameters }\end{array}$ & $\begin{array}{c}\text { PD-L } \\
\text { amplification }\end{array}$ & $\begin{array}{l}\text { PD-L1 } \\
\text { polysomy }\end{array}$ & $\begin{array}{l}\text { PD-L1 } \\
\text { disomy }\end{array}$ & $p$-value ${ }^{\mathrm{a}}$ & $p$-value ${ }^{b}$ \\
\hline Parametrial invasion & & & & 0.066 & $0.007^{\star}$ \\
\hline No & $0(0)$ & $5(10)$ & $20(31)$ & & \\
\hline Yes & $8(100)$ & $45(90)$ & $45(69)$ & & \\
\hline Lymph node positive & & & & $0.003^{*}$ & 0.153 \\
\hline No & $3(37)$ & $36(72)$ & $54(83)$ & & \\
\hline Yes & $5(63)$ & $14(28)$ & $11(17)$ & & \\
\hline Treatment & & & & 0.615 & 0.789 \\
\hline CTRT & $8(100)$ & $48(96)$ & $63(97)$ & & \\
\hline Radical RT & $0(0)$ & $2(4)$ & $2(3)$ & & \\
\hline
\end{tabular}

Values are presented as number (\%), mean \pm standard deviation.

a comparison between PD-L1 amplification and PD-L1 disomy.

${ }^{\mathrm{b}}$ comparison between PD-L1 polysomy and PD-L1 disomy.

Abbreviations: SCC, squamous cell carcinoma; Adeno, adenocarcinoma; CTRT, concurrent chemoradiotherapy; RT, radiation therapy. ${ }^{\star} p$-value $<0.05$, Statistically significant.

Patients with PD-L1 immunopositive tumours were associated with squamous cell carcinoma $(p<0.001)$ and had a significantly higher risk for parametrial invasion at diagnosis $(p<0.001)$, whereas PD-L1 immunohistochemical expression was not relevantly correlated with age, FIGO stage, tumour size or radio (chemo) therapy. In contrast, PD-L1 polysomy was associated with the presence of parametrial invasion $(p$ $=0.007)$ and FIGO stage III-IV $(p<0.001)$, whereas PD-L1 amplification was found to be relevantly correlated with nodal metastasis $(p=0.003)$. An increase in PD-L1 gene copy number was not correlated with age, histologic subtype, tumour size or radio (chemo) therapy.

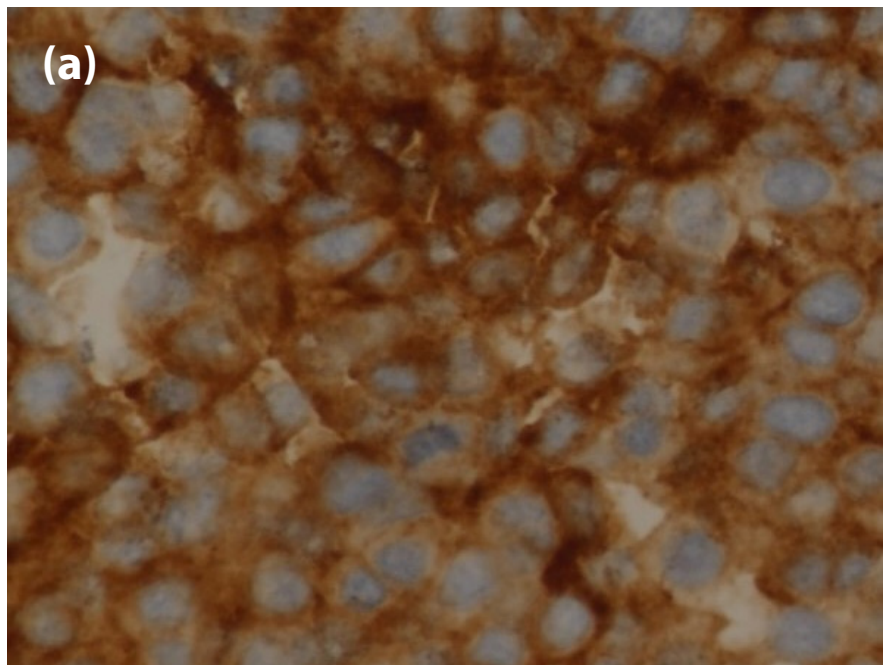

\section{Status of PD-L1 protein expression}

PD-L1 expression was evaluated in tumour cells of cervical carcinoma by immunohistochemistry. PD-L1 expression in at least $10 \%$ of tumour cells was identified in 103/123 (83\%) cervical carcinomas. The mean percentage of positive tumour cells (any strength of expression) was 25\% (range: 1\%-100\%). Strong, membranous staining (3+) was identified in $18 / 123$ (15\%) cases, moderate staining (2+) in $27 / 123$ (22\%) cases, and weak staining (1+) in 20/123 (16\%) cases (Figure 1a-d).

Figure 1. Representative pictures of PD-L1 IHC and FISH, showing (a) strong staining (3+), (b) moderate staining (2+), (c) weak staining $(1+)$, (d) negative staining (0), (e) PD-L1 amplification, (f) polysomy, and (g) disomy. The PD-L1 gene is labelled in green and centromere 9 in red. (a-d: original magnification $\times 60$, e-g: original magnification $\times 100$ ). 

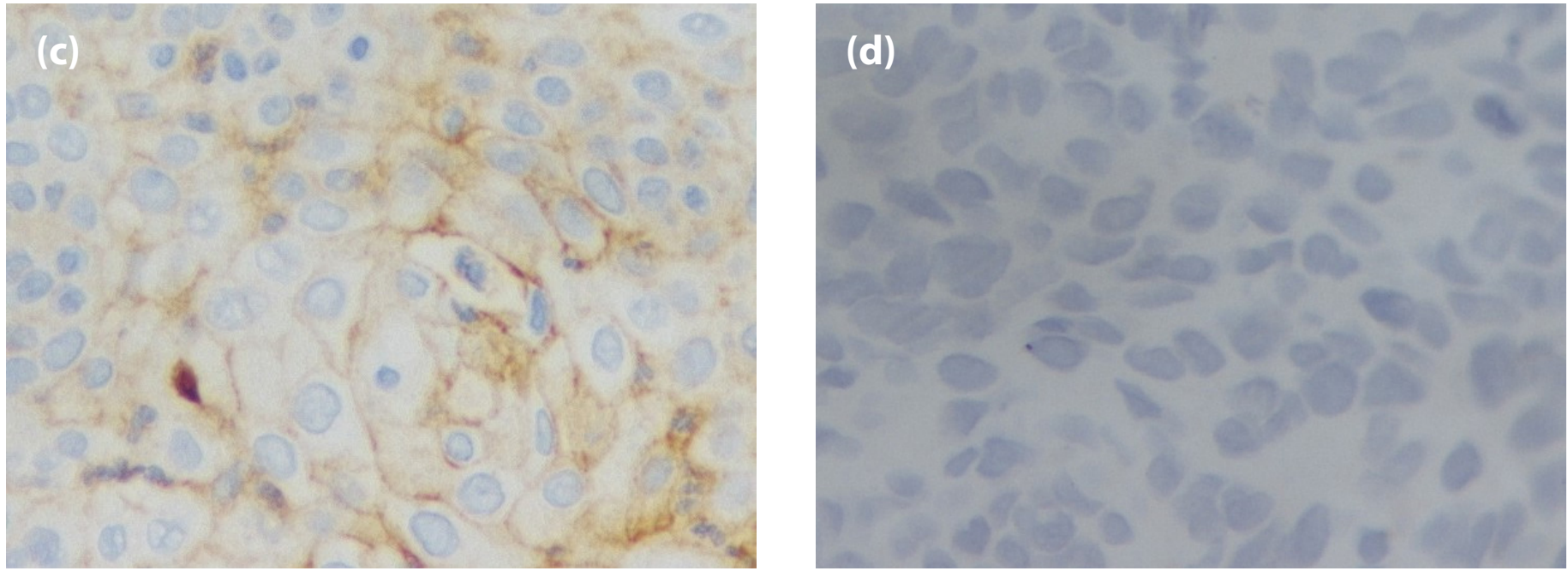

(e)

(f)
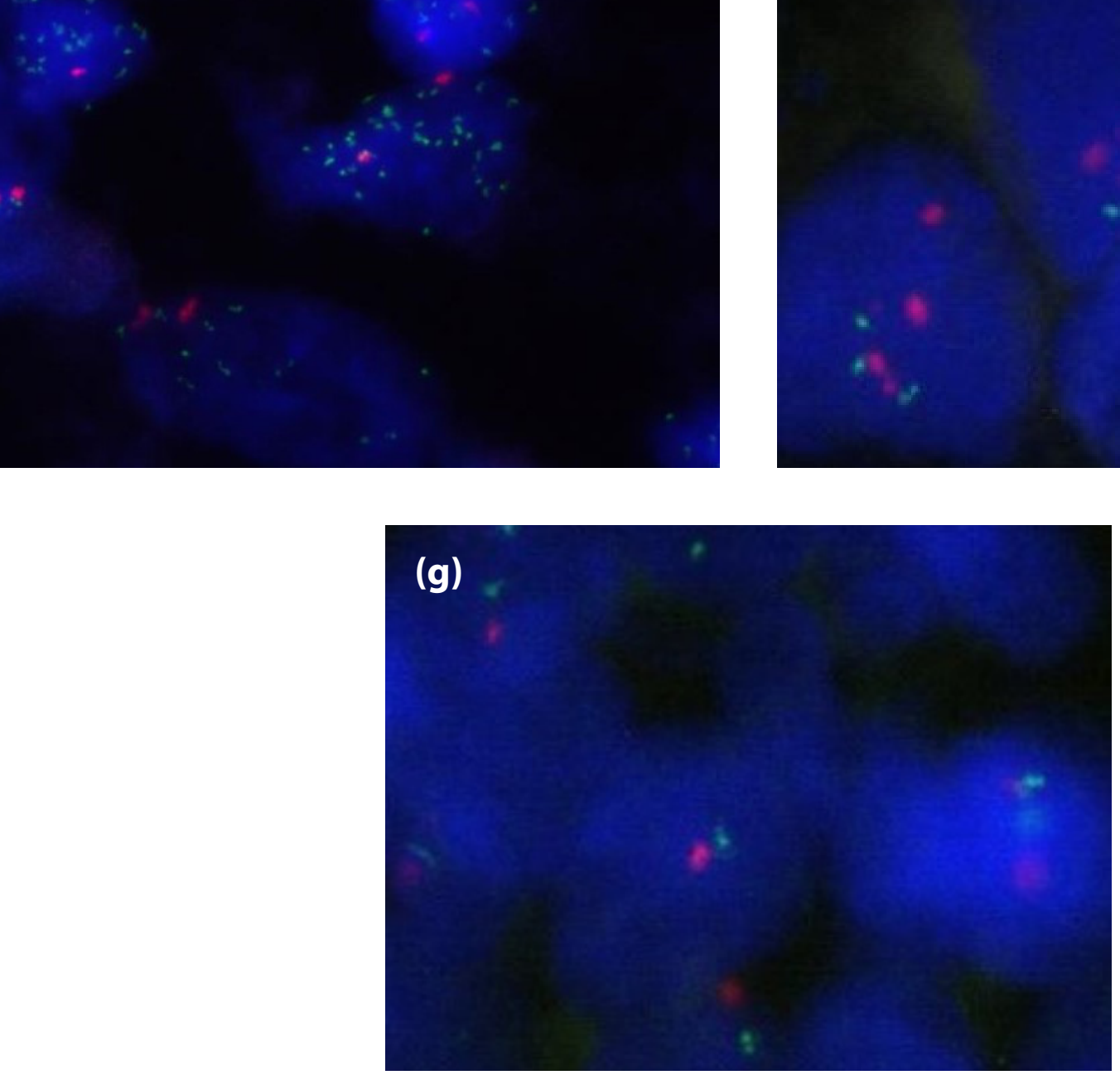

Figure 1. (Continued) 
Status of PD-L1 gene copy number alterations

PD-L1 amplification was identified in 8/123 (7\%) cases (Figure 1e). Gene copy number gain was confined to tumour cells and was not present in the white blood cell component. PD-L1 polysomy was observed in 50/123 (40\%) cases (Figure 1f). A total of $65 / 123$ (53\%) cases displayed a disomy (Figure 1g).

\section{Correlation between PD-L1 copy number gain and PD-L1 protein expression}

There was no association between PD-L1-positive tumours and the genetic category of the tumour $(p=0.169)$

The results for both PD-L1 IHC scores and PD-L1 FISH are shown in Table 3. Overall, tumours with PD-L1 gene amplification and polysomy displayed membranous PD-L1 immunostaining (scores $1+$ to $3+$ ) by immunohistochemistry in $7 / 8(88 \%)$ and $34 / 50(68 \%)$ cases, respectively. A significantly higher frequency of cases with PD-L1 amplification was PDL1 immunopositive (scores $1+$ to $3+$ ) than cases without amplification $(p=0.042)$. Likewise, the immunohistochemical expression of PD-L1 in tumours with PD-L1 polysomy was significantly higher than in tumours with disomy $(p<0.001)$. Moreover, 6/8 carcinoma cases with strong, membranous PDL1 immunostaining (score $3+$ ) showed PD-L1 amplification, $8 / 50$ showed a polysomy and 4/65 cases displayed a disomy. Interestingly, one of carcinoma cases with PD-L1 amplification was PD-L1 immunonegative (score 0). To confirm the findings and to exclude false negative staining due to tumour heterogeneity in this case, FISH analysis and immunohistochemistry were repeated on whole tissue sections. Likewise, whole sections of all carcinoma cases with PD-L1 immunopositivity (score $3+$ ) but negative results for amplification were also repeated, showing similar results.

(a)

PD-L1 Positive vs. PD-L1 Negative, $P=0.934$

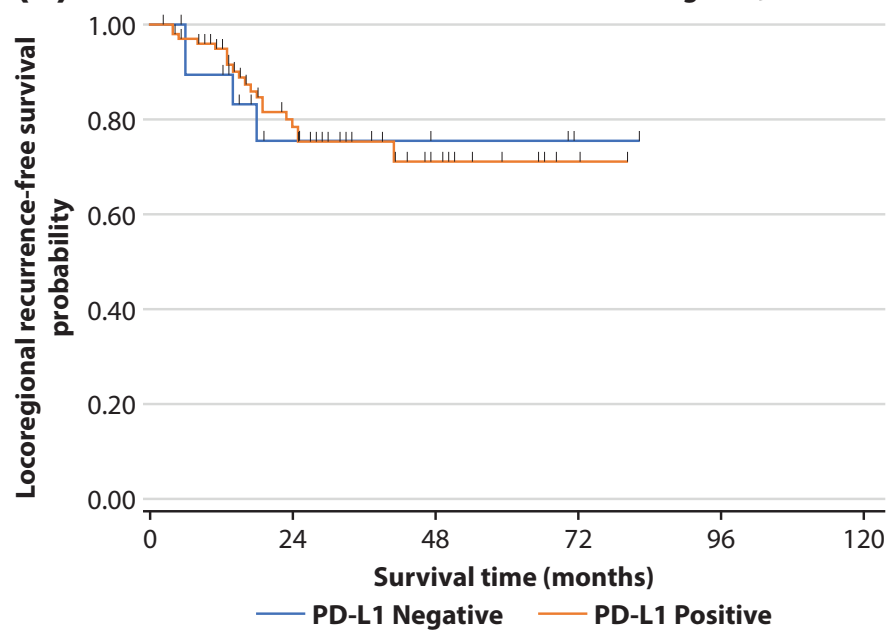

Table 3. PD-L1 FISH and PD-L1 immunohistochemistry.

\begin{tabular}{lccccc} 
& & \multicolumn{4}{c}{ PD-L1 IHC } \\
\cline { 3 - 6 } PD-L1 FISH & $\begin{array}{c}\text { Cases } \\
\mathbf{n}=123\end{array}$ & $\begin{array}{c}\text { Score } \\
3+\end{array}$ & $\begin{array}{c}\text { Score } \\
2+\end{array}$ & $\begin{array}{c}\text { Score } \\
1+\end{array}$ & $\begin{array}{c}\text { Score } \\
\mathbf{0}\end{array}$ \\
\hline Amplification & $8(7 \%)$ & $6 / 8$ & $1 / 8$ & $0 / 8$ & $1 / 8$ \\
Polysomy & $50(40 \%)$ & $8 / 50$ & $16 / 50$ & $10 / 50$ & $16 / 50$ \\
Disomy & $65(53 \%)$ & $4 / 65$ & $10 / 65$ & $10 / 65$ & $41 / 65$ \\
\hline
\end{tabular}

In further analyses, PD-L1 expression levels in tissues were evaluated using a modified $\mathrm{H}$ scoring system. The PD-L1 protein expression levels according to the copy number status of PD-L1. The mean $\mathrm{H}$ score in amplified tumours was significantly higher than in polysomic tumours and disomic tumours $(228 \pm 95,62 \pm 53$, and $16 \pm 14$, respectively; $p<$ 0.001).

\section{Survival outcomes}

Figure 2 shows the Kaplan-Meier survival curves for cervical cancer patients, according to the IHC-based (Figure 2ac) and FISH-based (Figure 2d-f) expression status of PD-L1 in tumours. The results of univariate and multivariate analyses evaluating the impact of various known prognostic factors on LRR, RFS and CSS are summarized in Table 4. Overall, FIGO stage, tumour size, number of metastatic lymph nodes and PD-L1 amplification were univariately associated with RFS and CSS. Nevertheless, on multivariate analysis, FIGO stage continued to show a significant impact on RFS (HR, 2.22; 95\%CI, 1.17-4.20; $p=0.015$ ) and CSS (HR, 13.25; 95\%CI, 4.45-39.45; $p<0.001)$, whereas PD-L1 amplification showed a significant impact on RFS on multivariate analysis (HR, 5.68; 95\%CI, 1.98-16.28; $p=0.001)$. Only PD-L1 polysomy showed a significant impact on LRR in both univariate and multivariate analyses (HR, 4.13; 95\%CI, 1.63-10.49; $p=0.003)$.

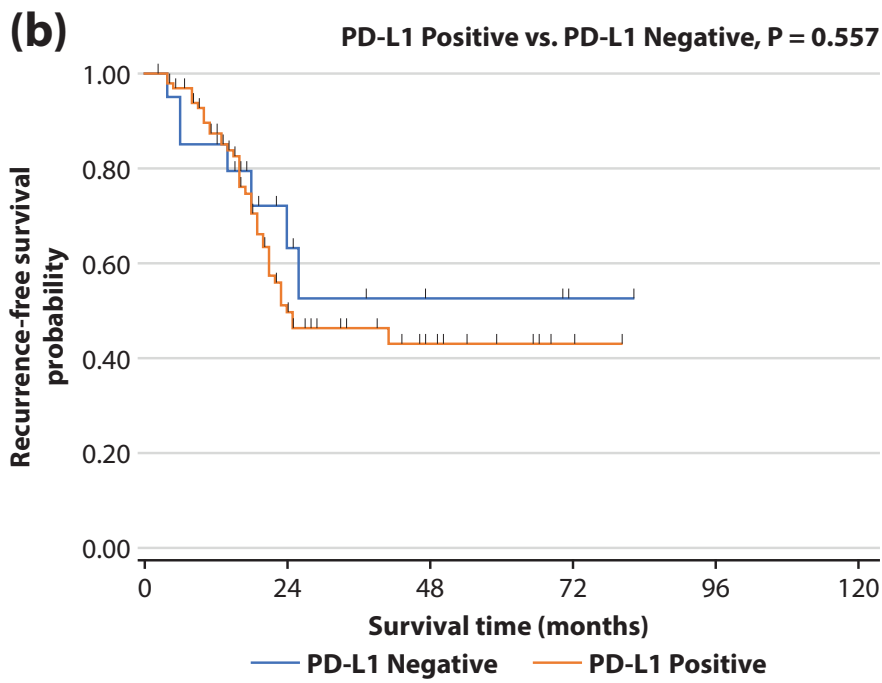

Figure 2. Kaplan-Meier survival curves for cervical cancer patients in relation to PD-L1 immunoreactivity (Figure 2a-c) and genetic category (amplification, polysomy, and disomy) (Figure 2d-f) in regard to LRR, RFS, and CSS. LRR, locoregional recurrence-free survival; RFS, recurrence-free survival; CSS, cancer-specific survival. 
(c)

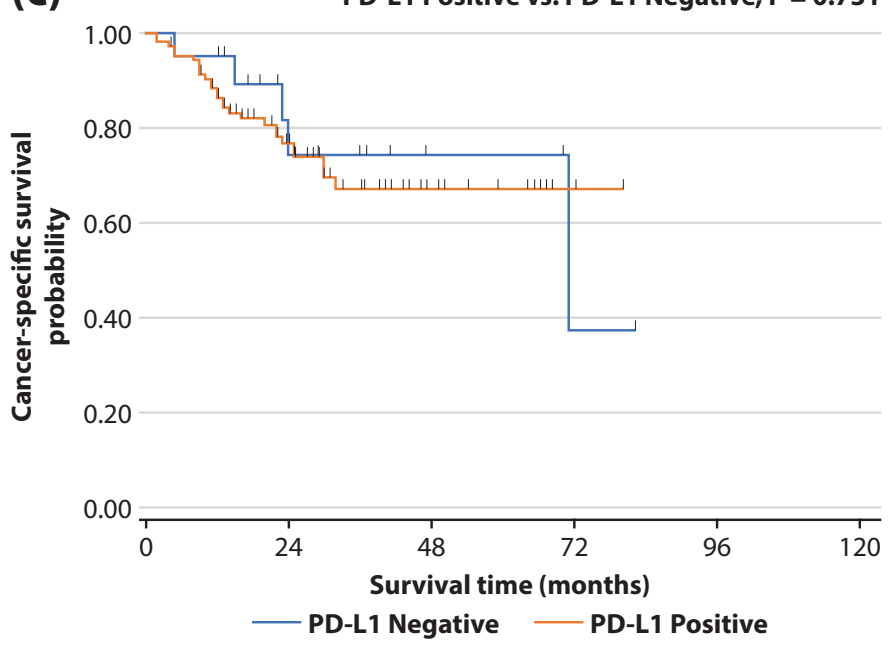

PD-L1 disomy vs. PD-L1 polysomy, $P=0.002$
PD-L1 disomy vs. PD-L1 amplification, $P<0.001$

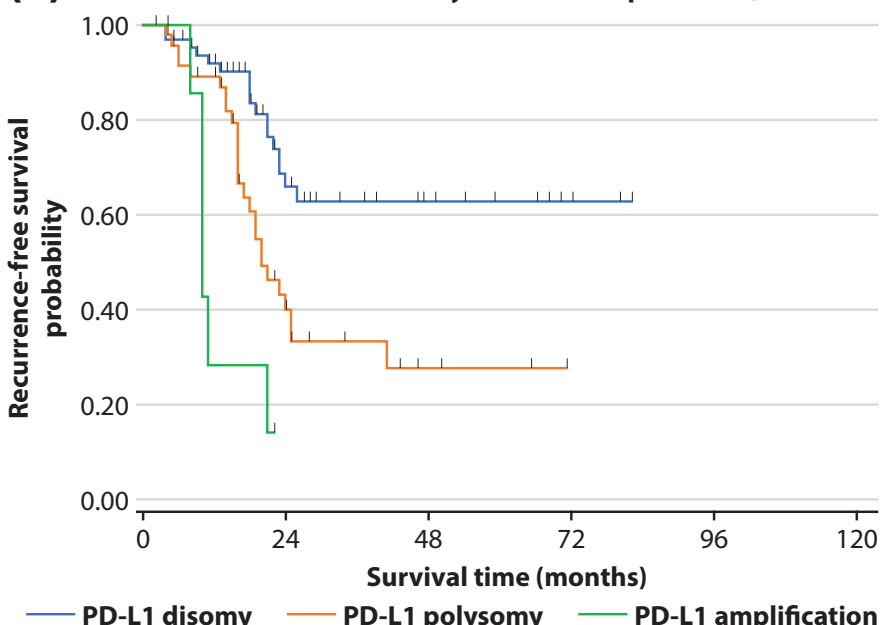

PD-L1 disomy vs. PD-L1 polysomy, $P=0.001$ (d) PD-L1 disomy vs. PD-L1 amplification, $P=0.552$

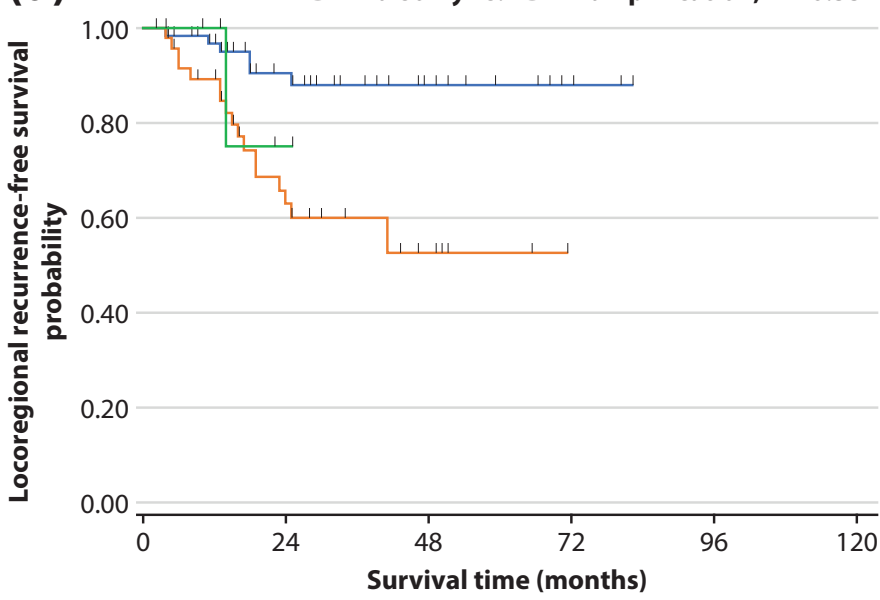

— PD-L1 disomy _ PD-L1 polysomy — PD-L1 amplification

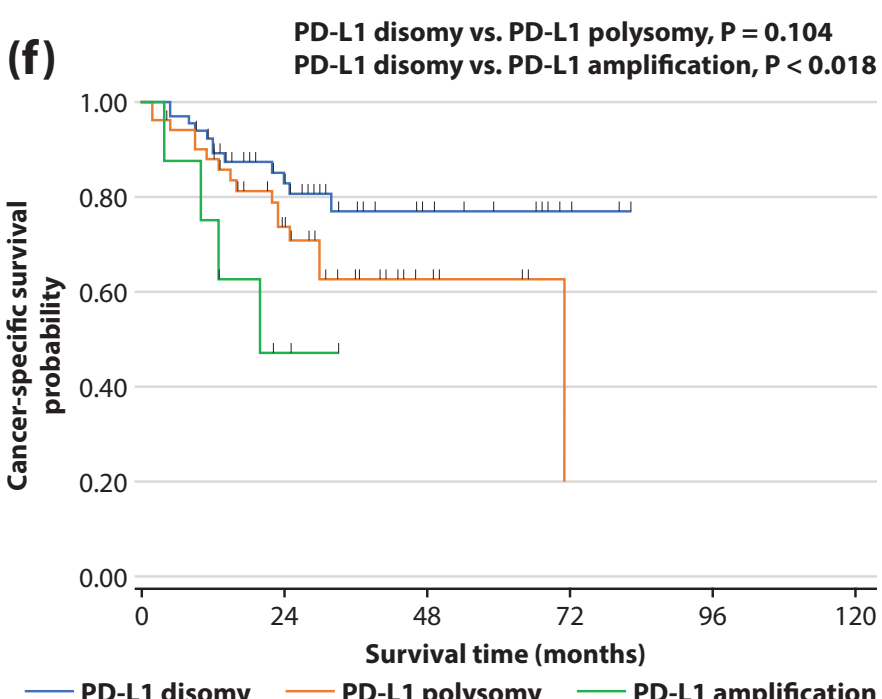

Figure 2. (Continued)

Table 4. Univariate and Multivariate survival analysis. $(n=123)$

\begin{tabular}{|c|c|c|c|c|c|c|c|}
\hline \multirow{2}{*}{ Variables } & \multirow{2}{*}{$\begin{array}{c}\text { Number } \\
\text { (n) }\end{array}$} & \multicolumn{3}{|c|}{ Univariate analysis ( $p$-value) } & \multicolumn{3}{|c|}{ Multivariate analysis ( $p$-value) } \\
\hline & & LRR & RFS & CSS & LRR & RFS & CSS \\
\hline \multicolumn{8}{|l|}{ Age (years) } \\
\hline$<60$ Vs. $\geq 60$ & 81 Vs. 42 & 0.229 & 0.202 & 0.577 & & & \\
\hline \multicolumn{8}{|l|}{ Histology } \\
\hline SCC Vs. Adeno & 110 Vs. 13 & 0.115 & 0.580 & 0.690 & & & \\
\hline \multicolumn{8}{|l|}{ Tumor size $(\mathrm{cm})$} \\
\hline$<4$ Vs. $\geq 4$ & 30 Vs. 93 & 0.4 & $0.016^{*}$ & $0.020^{*}$ & & 0.453 & 0.221 \\
\hline \multicolumn{8}{|l|}{ FIGO stage } \\
\hline Stage IB2-IIB Vs. Stage IIIA-IVA & 77 Vs. 46 & 0.163 & $0.001^{\star}$ & $<0.001^{*}$ & & $0.015^{*}$ & $<0.001^{*}$ \\
\hline \multicolumn{8}{|l|}{ Parametrial invasion } \\
\hline Absent Vs. Present & 25 Vs. 98 & 0.985 & 0.198 & & & & \\
\hline
\end{tabular}


Table 4. (Continued)

\begin{tabular}{|c|c|c|c|c|c|c|c|}
\hline \multirow{2}{*}{ Variables } & \multirow{2}{*}{$\begin{array}{c}\text { Number } \\
\text { (n) }\end{array}$} & \multicolumn{3}{|c|}{ Univariate analysis ( $p$-value) } & \multicolumn{3}{|c|}{ Multivariate analysis ( $p$-value) } \\
\hline & & LRR & RFS & CSS & LRR & RFS & CSS \\
\hline \multicolumn{8}{|l|}{ Metastatic lymph node } \\
\hline Absent Vs. Present & 93 Vs. 30 & 0.122 & $0.022^{*}$ & $0.009^{*}$ & & 0.509 & 0.143 \\
\hline \multicolumn{8}{|l|}{ Treatment } \\
\hline CTRT Vs. Radical RT & 119 Vs. 4 & & & & & & \\
\hline \multicolumn{8}{|l|}{ PD L1 expression } \\
\hline Negative Vs. Positive & 20 Vs.103 & 0.934 & 0.563 & 0.732 & & & \\
\hline \multicolumn{8}{|l|}{ PD L1 copy number alterations } \\
\hline Polysomy Vs. Disomy & 50 Vs. 65 & $0.003^{*}$ & $0.003^{\star}$ & 0.112 & $0.003^{*}$ & 0.138 & 0.377 \\
\hline Amplification Vs. Disomy & 8 Vs. 65 & 0.524 & $<0.001^{*}$ & $0.018^{*}$ & 0.524 & $0.001^{*}$ & 0.221 \\
\hline
\end{tabular}

Abbreviations: LRR, locoregional recurrence; RFS, recurrence-free survival; CSS, cancer-specific survival; SCC, squamous cell carcinoma; Adeno, adenocarcino$\mathrm{ma}$; CTRT, concurrent chemoradiotherapy; RT, radiation therapy; ${ }^{*} p$-value $<0.05$, Statistically significant.

\section{Discussion}

Cancer cells exert various methods of immune inactivation to oppose anticancer immunity. One of these methods is the modification of the PD-1/PD-L1 pathway, which is known as the immune checkpoint. ${ }^{21}$ The $\mathrm{PD}-1 / \mathrm{PD}-\mathrm{L} 1$ pathway not only normally regulates exaggerated immune responses but also appears to be a route exploited by cancer cells to escape the immune system. ${ }^{22}$ The activation of this pathway can give rise to cancer immune evasion and facilitate cancer cell proliferation through mechanisms including $\mathrm{T}$ cell tolerance, $\mathrm{T}$ cell exhaustion, $\mathrm{T}$ cell apoptosis, enhancement of immunosuppressive Treg cell function and PD-1 disbalance. ${ }^{23}$ Clinically, expression of PD-L1 in cancer cells is thought to be predictive of tumour response to immunomodulatory therapies targeting the PD-1/PD-L1 pathway. ${ }^{18}$

PD-L1 expression has been identified in many solid cancers, ${ }^{10}$ such as malignant melanoma, ${ }^{11}$ pulmonary cancer ${ }^{12}$ and colorectal cancer. ${ }^{13}$ However, data on PD-1 expression in cervical carcinoma regarding prevalence, prognostic impact and variation of expression in disease course is limited. In this study, our results showed that the amplification of the PD-L1 gene can be identified in a subset of cervical carcinomas and that gene amplification is correlated with expression levels of the PD-L1 protein in the majority of amplified cases.

Using immunohistochemistry and a cut-off point for positivity defined as at least $10 \%$ of malignant cells showing membranous PD-L1 staining, we identified PD-L1 expression in $83 \%$ of cervical carcinomas, a relatively higher proportion than in previous studies. ${ }^{24-27}$ To date, only a few studies have investigated PD-L1 expression in cervical carcinoma with positivity rates ranging between $30 \%-70 \% .^{24-27}$ This wide range of detection rates is likely due to several factors: (1) the use of different scoring methods and cut-off points for the definition of positivity; (2) the use of different antibody clones for immunohistochemistry-the SP263 clone used in our study might have a superior sensitivity compared to the other clones due to the number of positively stained cells and its staining intensity; ${ }^{28-30}$ (3) the condition of tissue fixation and stability of epitopes during immunohistochemistry reactions; and (4) the heterogeneity of PD-L1 staining in different areas of the tumour.

Theoretically, the expression of PD-L1 in malignant cells is regulated by different pathways. An in vitro study in cultured SCCHN cell lines demonstrated that PD-L1 expression is significantly upregulated in response to interferon gamma $($ IFN- $\gamma$ ), an important cytokine that triggers de novo PD-L1 induction in malignant cells as well as in normal tissues. ${ }^{31}$ The expression of PD-L1 in malignant cells is controlled by a variety of intracellular signalling pathways. PD-L1 expression can be stimulated by autocrine/paracrine mediators within the cancer microenvironment, especially IFN- $\gamma$. The interaction between extrinsic stimuli and the IFN- $\gamma$ receptor could lead to the expression and activation of various downstream signalling pathways, including NF-kB, MAPK, PI3K, mTOR and JAK/STAT, that promote cell cycle progression and the activation of transcription factors. Such signalling pathways further regulate the nuclear translocation of transcription factors to the PD-L1 promoter. ${ }^{32}$

Nevertheless, the expression of PD-L1 can fluctuate at different times during the disease course. In contrast, IFN- $\gamma-$ induced PD-L1 expression can be continuously activated via gene amplification events involving the gene locus on chromosome 9p24.1. The additional somatic copy number alterations resulting in an increase of the fraction of DNA regions could be associated with carcinogenesis and cancer progression.

Several important genes are known to be amplified and have been identified as prognostic markers, mechanisms of drug resistance, or treatment targets in some cancers, such as non-small-cell lung cancer. ${ }^{33}$ The 9p24.1 chromosomal locus contains the PD-L1, PD-L2 and JAK2 genes. Selective 9p24.1 amplification has been recently recognized as a key mechanism for increased PD-L1 expression in nodular sclerosis, classical Hodgkin's lymphoma and primary mediastinal large $\mathrm{B}$-cell lymphoma ${ }^{14}$ and has also been identified 
in a subset of colorectal carcinomas, triple-negative breast cancers, glioblastomas, gastric adenocarcinomas, ${ }^{15,16}$ and cervical and vulvar carcinomas. ${ }^{17}$

In the present study, our results demonstrated that PDL1 copy number gain (amplification and polysomy) can be observed in a subset of cervical cancer patients using FISH analysis (47\% of cervical cancer cases). Notably, gene copy number gain was confined to tumour cells and was not present in tumour-infiltrating immune cells. The results indicated that PD-L1 expression on tumour cells could be regulated by tumour-intrinsic mechanisms involving gene copy number gains, whereas PDL1 expression on immune cells was more likely associated with IFN- $\gamma$-induced adaptive regulation.

In addition, we found that PD-L1 copy number gain was superior to PD-L1 immunohistochemical expression and could act as an independent and strong predictor of survival outcomes in cervical carcinoma. The amplification-driven PD-L1 expression in a subgroup of cervical carcinomas may demonstrate a new subgroup of cervical cancer with a disease-specific genetic alteration. Further studies are required to evaluate the impact of PD-L1 amplification on pathogenesis and disease progression and on the prognosis of this newly recognized subgroup of cervical carcinomas.

In the case of PD-L polysomy, the data we gathered indicated that the survival rate for patients with polysomy lay between those for disomy and for amplification. These findings showed that it is possible to further categorize PD-L polysomy as high or low polysomy. For low polysomy, the prognosis was similar to that of disomy, while the prognosis for high polysomy more closely followed that of amplification. However, it is necessary to further validate the optimal cut-off values for these polysomy subtypes. In contrast to the previous study, ${ }^{17}$ our results showed that PD-L1 amplification can be identified in only a minority of cases (7\% of cases). These conflicting data can be explained by differences in sample size, disease stage, or underlying diseases in the studied population.

In this study, it was interesting to note that one of the amplified carcinoma cases was PD-L1 immunonegative (score 0). It is not yet clear why this discrepancy arose, although it is possible that technical difficulties occurring with the antibody clone might be responsible; alternatively, the reason may be linked to posttranscriptional or posttranslational modifications.

Blocking the PD-1/PD-L1 axis could be a promising treatment option in several tumour types, such as malignant melanoma and non-small-cell lung cancer, as well as cervical cancer. These studies have shown better response rates in patients with high PD-L1 expression. However, immune checkpoint blockage has shown remarkable response rates in lymphomas, particularly Hodgkin's lymphoma, even with PD-L1 amplification as well. ${ }^{34}$ The identification of PD-L1 gene copy number gain as a powerful mechanism for PD-L1 expression in the present study may provide a rationale for the treatment of cervical cancer patients, particularly in a subgroup of cervical cancer with PDL1 gene amplification. Future studies will need to investigate whether PD-L1 copy number status might be a better predictive molecular biomarker for tumour response to blockage of the PD-1/PD-L1 pathway than PD-L1 immunohistochemical status.

\section{Conclusions}

PD-L1 copy number increase was associated with PD-L1 protein expression and was demonstrated to be a strong and independent factor of poor survival outcomes in patients with cervical cancer. An increase in PD-L1 gene copy number can be an alternative biomarker for predicting the response to anti-PD-1/PD-L1 therapy in cervical cancer patients. The identification of PD-L1 gene copy number gain as a potential mechanism for PD-L1 overexpression in the present study may provide a rationale for the treatment of cervical cancer patients in this subgroup as well. Further research is needed to examine whether PD-L1 copy number alterations are clinically associated with a benefit of anti-PD-1/PD-L1 treatment in cervical cancer patients.

\section{Acknowledgments}

The authors would like to thank the following colleagues at Department of Anatomical Pathology, Navamindradhiraj University: Dr. Somneuk Jesadapatarakul and Mrs. Pornpimon Kongjan for psychological support and theoretical guidance during the present study. The authors are particularly grateful to Dr. Thawi Yingsa-nga (Division of Nuclear Medicine, Maharat Nakhon Ratchasima Hospital) and Dr. Wanniga Saengsuri (Division of Gynecologic Oncology, Taksin Hospital) for clinical advice during the present study. Finally, the authors would like to express their thanks to Ms. Oraphan Wanacharoen (BCC MDx Co., Ltd., Bangkok, Thailand) for her kind technical help.

\section{Competing interests}

The authors declare that they have no conflict of interest.

\section{References}

1. Parkin DM, Bray F, Ferlay J, Pisani P. Global cancer statistics, 2002. CA Cancer J Clin. 2005;55:74-108.

2. Mohar A, Frías-Mendívil M. Epidemiology of cervical cancer. Cancer Invest. 2000;18:584-90.

3. Hong JH, Tsai CS, Lai CH, Chang TC, Wang CC, Chou HH, et al. Recurrent squamous cell carcinoma of cervix after definitive radiotherapy. Int J Radiat Oncol Biol Phys. 2004;60(1):249-57.

4. Perez CA, Grigsby PW, Camel HM, Galakatos AE, Mutch D, Lockett MA. Irradiation alone or combined with surgery in stage IB, IIA, and IIB carcinoma of the uterine cervix: update of a nonrandomized comparison. Int J Radiat Oncol Biol Phys. 1995;31:703-16.

5. Dong H, Strome SE, Salomao DR, Tamura H, Hirano F, Flies DB, et al. Tumor-associated B7-H1 promotes T-cell apoptosis: a potential mechanism of immune evasion. Nat Med. 2002;8:793-800.

6. Barber DL, Wherry EJ, Masopust D, Zhu B, Allison JP, Sharpe AH, et al. Restoring function in exhausted CD8 T cells during chronic viral infection. Nature. 2006;439:682-7.

7. Yokosuka T, Takamatsu M, Kobayashi-Imanishi W, Hashimoto-Tane A, Azuma M, Saito T. Programmed cell death 1 forms negative costimulatory microclusters that directly inhibit $\mathrm{T}$ cell receptor signaling by recruiting phosphatase SHP2. J Exp Med. 2012;209:1201-17.

8. Latchman Y, Wood CR, Chernova T, Chaudhary D, Borde M, Chernova I, et al. PD-L2 is a second ligand for PD-1 and inhibits T cell activation. Nat Immunol. 2001;2:261-8.

9. Chinai JM, Janakiram M, Chen F, Chen W, Kaplan M, Zang X. New immunotherapies targeting the PD-1 pathway. Trends Pharmacol Sci. 2015. 36:587-95.

10. Wu P, Wu D, Li L, Chai Y, Huang J. PD-L1 and Survival in Solid Tumors: A Meta-Analysis. PLoS One. 2015; 10:e0131403. 
11. Hino R, Kabashima K, Kato Y, Yagi H, Nakamura M, Honjo T, et al. Tumor cell expression of programmed cell death-1 ligand 1 is a prognostic factor for malignant melanoma. Cancer. 2010; 116:1757-66.

12. Velcheti V, Schalper KA, Carvajal DE, Anagnostou VK, Syrigos KN, Sznol $\mathrm{M}$, et al. Programmed death ligand-1 expression in non-small cell lung cancer. Lab Invest. 2014; 94:107-16.

13. Shi SJ, Wang LJ, Wang GD, Guo ZY, Wei M, Meng YL, et al. B7-H1 expression is associated with poor prognosis in colorectal carcinoma and regulates the proliferation and invasion of HCT116 colorectal cancer cells. PLoS One. 2013; 8:e76012.

14. Green MR, Monti S, Rodig SJ, Juszczynski P, Currie T, O’Donnell E, et al. Integrative analysis reveals selective 9p24.1 amplification, increased PD-1 ligand expression, and further induction via JAK2 in nodular sclerosing Hodgkin lymphoma and primary mediastinal large B-cell lymphoma. Blood. 2010; 116:3268-77.

15. Barrett MT, Anderson KS, Lenkiewicz E, Andreozzi M, Cunliffe HE, Klassen CL, et al. Genomic amplification of 9p24.1 targeting JAK2, PD-L1, and PD-L2 is enriched in high-risk triple negative breast cancer. Oncotarget. 2015; 6: 26483-93.

16. Cancer Genome Atlas Research N. Comprehensive molecular characterization of gastric adenocarcinoma. Nature. 2014; 513: 202-9.

17. Howitt BE, Sun HH, Roemer MG, Kelley A, Chapuy B, Aviki E, et al. Genetic basis for PD-L1 expression in squamous cell carcinomas of the cervix and vulva. JAMA Oncol. 2016; 2: 518-22.

18. Hofmann M, Stoss O, Shi D, Buttner R, van de Vijver M, Kim W, et al. Assessment of a HER2 scoring system for gastric cancer: results from a validation study. Histopathology. 2008; 52: 797-805.

19. Villaruz LC, Ancevski Hunter K, Kurland BF, Abberbock S, Herbst C, Dacic S. Comparison of PD-L1 immunohistochemistry assays and response to $\mathrm{PD}-1 / \mathrm{L} 1$ inhibitors advanced non-small-cell lung cancer in clinical practice. Histopathology. 2019; 74: 269-75.

20. Inoue Y, Yoshimura K, Mori K, Kurabe N, Kahyo T, Mori H, et al. Clinical significance of PD-L1 and PD-L2 copy number gains in non-small-cell lung cancer. Oncotarget. 2016;7:32113-28.

21. Hamanishi J, Konishi I. Targeting the PD-1/PD-L1 immune checkpoint signal - a new treatment strategy for cancer. Gan To Kagaku Ryoho. 2014;41:1071-6.

22. Mahoney KM, Freeman GJ, McDermott DF. The Next Immune -Checkpoint Inhibitors: PD-1/PD-L1 Blockade in Melanoma. Clin Ther. 2015;37:764-82.
23. Zhang J, Gao J, Li Y, Nie J, Dai L, Hu W, et al. Circulating PD-L1 in NSCLC patients and the correlation between the level of PD-L1 expression and the clinical characteristics. Thorac Cancer. 2015;6:534-8.

24. Mezache L, Paniccia B, Nyinawabera A, Nuovo GJ. Enhanced expression of PD L1 in cervical intraepithelial neoplasia and cervical cancers. Mod Pathol. 2015;28: 1594-602.

25. Heeren AM, Punt S, Bleeker MC, Gaarenstroom KN, van der Velden J, Kenter GG, et al. Prognostic effect of different PD-L1 expression patterns in squamous cell carcinoma and adenocarcinoma of the cervix. Mod Pathol. 2016; 29: 753-63.

26. Reddy OL, Shintaku PI, Moatamed NA. Programmed death-ligand 1 (PD-L1) is expressed in a significant number of the uterine cervical carcinomas. Diagn Pathol. 2017;12: 45.

27. Meng Y, Liang H, Hu J, Liu S, Hao X, Wong MSK, et al. PD-L1 expression correlates with tumor infiltrating lymphocytes and response to neoadjuvant chemotherapy in cervical cancer. J Cancer. 2018;9(16):2938-45.

28. Smith J, Robida MD, Acosta K, Vennapusa B, Mistry A, Martin G, et al Quantitative and qualitative characterization of two PD-L1 clones: SP263 and E1L3N. Diagn Pathol. 2016;11:44.

29. Parra ER, Villalobos P, Mino B, Rodriguez-Canales J. Comparison of different antibody clones for immunohistochemistry detection of programmed cell death ligand 1 (PD-L1) on non-small cell lung carcinoma. Appl Immunohistochem Mol Morphol. 2018;26:83-93.

30. Munari E, Rossi G, Zamboni G, Lunardi G, Marconi M, Sommaggio $\mathrm{M}$, et al. PD-L1 assays 22C3 and SP263 are not interchangeable in non-small cell lung cancer when considering clinically relevant cutoffs: an interclone evaluation by differently trained pathologists. Am J Surg Pathol. 2018;42:1384-9.

31. Tsushima F, Tanaka K, Otsuki N, Youngnak P, Iwai H, Omura K, et al. Predominant expression of $\mathrm{B} 7-\mathrm{H} 1$ and its immunoregulatory roles in oral squamous cell carcinoma. Oral Oncol. 2006;42(3):268-74.

32. Ritprajak P, Azuma M. Intrinsic and extrinsic control of expression of the immunoregulatory molecule pd-11 in epithelial cells and squamous cell carcinoma. Oral Oncol. 2015;51:221-8.

33. Inoue $\mathrm{Y}$, Matsuura $\mathrm{S}$, Kurabe $\mathrm{N}$, Kahyo $\mathrm{T}$, Mori $\mathrm{H}$, Kawase A, et al Clinicopathological and survival analysis of Japanese patients with resected non-small-cell lung cancer harboring NKX2-1, SETDB1, MET, HER2, SOX2, FGFR1, or PIK3CA gene amplification. J Thorac Oncol. 2015; 10:1590-600.

34. Kline J, Bishop MR. Update on checkpoint blockade therapy for lymphoma. J Immunother Cancer. 2015; 3:33. 